\title{
Variação na prevalência de risco nutricional em indivíduos hospitalizados conforme cinco protocolos de triagem nutricional
}

\author{
Variation in the prevalence of nutritional risk in hospitalized individuals according to \\ five nutritional screening protocols
}

\author{
Juliane Pereira Duarte1, Juliana Paludo², Joana Raquel Nunes Lemos ${ }^{3}$, Thaís Rodrigues Moreira ${ }^{4}$ \\ ${ }^{1}$ Discente do curso de Nutrição do Centro Universitário Univates, Lajeado, RS. ${ }^{2}$ Nutricionista, Mestre em Saúde da Criança e do Adolescente pela Universidade Federal \\ do Rio Grande do Sul (UFRGS), Porto Alegre, RS; ${ }^{3}$ Nutricionista graduada pela Universidade do Vale do Rio dos Sinos. Mestre em Ciências Médicas pela UFRGS, Docente \\ do Centro Universitário Ritter dos Reis, Porto Alegre, RS; ${ }^{4}$ Nutricionista graduada pelo Centro Universitário Franciscano, Mestre em Ciências Médicas pela UFRGS,
} Docente do curso de Nutrição do Centro Universitário Univates, Porto Alegre, RS.

\section{RESUMO}

Objetivos: Verificar a prevalência de risco nutricional em indivíduos hospitalizados e a concordância no diagnóstico nutricional através de cinco protocolos de triagem nutricional.

Métodos: Um estudo transversal incluiu pacientes de ambos os gêneros, maiores de 18 anos, admitidos no hospital no período de janeiro a março de 2013. Dentro das primeiras 48 horas de internação, foram aplicados os seguintes protocolos de triagem nutricional: Mini Nutrition Assessment (MNA); Mini Nutrition Assessment Short Form (MNA-SF); Subjective Global Assessment (ASG); Nutritional Risk Screening (NRS 2002); e Malnutrition Universal Screening Tool (MUST). Os protocolos são compostos de questões relacionadas à perda de peso, índice de massa corporal, capacidade funcional, inapetência alimentar e alterações gastrintestinais. Os indivíduos foram classificados em dois níveis: bem nutridos e desnutridos.

Resultados: Foi avaliada uma amostra de 100 pacientes, que evidenciou 49 (49\%) indivíduos com risco nutricional pelo protocolo MNA; 53 (53\%) pelo MNA-SF; 23 (23\%) pelo MUST; 7 (7\%) pelo NRS; e 4 (4\%) pelo ASG. O gênero feminino e idades superiores a 60 anos prevaleceram na amostra. Três dos protocolos, MNA-SF, MUST e NRS, detectaram maior risco nutricional em pacientes com mais de 60 anos $(\mathrm{p} \leq 0,05)$. Observou-se que MNA e MNA-SF foram os protocolos que obtiveram melhor concordância (coeficiente Kappa=0,760, $\mathrm{p}<0,001$ ). Conclusões: Houve boa concordância entre os protocolos de MNA e MNA-SF, porém não destes com os demais protocolos, que também não apresentaram concordância entre si. O protocolo que classificou a maior porcentagem de pacientes com risco nutricional foi o MNA-SF, e o que menos detectou risco nutricional foi o ASG.

DESCRITORES: AVALIAÇÃO NUTRICIONAL; DESNUTRIÇÃO PROTEICO-CALÓRICA; ADULTO; IDOSO.

\section{ABSTRACT}

Aims: To assess the prevalence of nutritional risk in hospitalized subjects and the agreement in nutritional diagnosis between five nutritional screening protocols.

Methods: A cross-sectional study included patients of both genders, aged 18, admitted to hospital from January to March 2013 Within the first 48 hours of admission, the following nutritional screening protocols were applied: Mini Nutrition Assessment (MNA); Mini Nutrition Assessment Short Form (MNA-S); Subjective Global Assessment (ASG); Nutritional Risk Screening (NRS 2002); and Malnutrition Universal Screening Tool (MUST). The protocols consist of questions related to weight loss, body mass index, functional capacity, food appetite and gastrointestinal disturbances. Subjects were classified into two levels: well-nourished and malnourished.

Results: A sample of 100 patients were evaluated, which showed 49 (49\%) individuals at nutritional risk by the MNA protocol; 53 (53\%) by MNA-SF; 23 (23\%) by MUST; 7 (7\%) by NRS; and 4 (4\%) by ASG. The female and aged over 60 years prevailed in the sample. Three protocols, MNA-SF, MUST and NRS, showed a higher nutritional risk in patients over 60 years ( $\mathrm{p} \leq 0.05)$. It was observed that MNA and MNA-SF protocols obtained better agreement (Kappa coefficient $=0.760, \mathrm{p}<0.001)$.

Conclusions: There was good agreement between the protocols MNA and MNA-SF, but not of those with other protocols, which also showed no correlation with each other. The protocol that ranked the highest percentage of patients at nutritional risk was the MNA-SF, and less nutritional risk was detected by ASG.

KEY WORDS: NUTRITION ASSESSMENT; PROTEIN-ENERGY MALNUTRITION; ADULT; AGED. 


\section{INTRODUÇÃO}

O estudo da desnutrição no âmbito hospitalar tem sido destaque nos últimos anos. Evidências científicas mostram que a desnutrição está associada ao aumento da morbidade e mortalidade dos indivíduos. ${ }^{1}$ No Brasil, segundo o Inquérito Brasileiro de Avaliação Hospitalar (IBRANUTRI), realizado com 4.000 pacientes em 25 hospitais públicos de diversas regiões do país, a desnutrição estava presente em $48,1 \%$ dos pacientes internados, sendo que $12,5 \%$ apresentavam desnutrição grave e $31,8 \%$ encontravam-se desnutridos nas primeiras 48 horas de internação. ${ }^{2}$

Diante disso, em 2005, o Ministério da Saúde instituiu, através da Portaria $n^{\circ} 343$, a obrigatoriedade de protocolos de triagem nutricional nos hospitais, para a avaliação do risco nutricional. ${ }^{3}$ Conforme estudo de Beck et al, ${ }^{4}$ os motivos pelos quais a prática da triagem nutricional não era aplicada eram falta de conhecimento, instruções e treinamentos nos métodos utilizados. Em nível internacional, estudo realizado pela European Society of Parenteral and Enteral Nutrition (ESPEN) orienta que todos os hospitais desenvolvam políticas e protocolos específicos para identificar o risco nutricional em indivíduos hospitalizados, no intuito de um tratamento mais rápido e eficaz. ${ }^{5}$

Sabe-se que a desnutrição está diretamente associada à incidência de infecções clínicas, desfavorecendo a evolução clínica do indivíduo e ocasionando aumento do período de hospitalização. ${ }^{6}$ Nesse contexto, torna-se de grande importância avaliar o estado nutricional dos indivíduos hospitalizados para que a terapia nutricional possa ser iniciada, visando colaborar no tratamento e na prevenção da desnutrição.?

Para a avaliação nutricional de indivíduos hospitalizados podem-se utilizar múltiplos parâmetros, porém destacam-se os protocolos de triagem nutricional, que objetivam a identificação de pacientes desnutridos ou com risco nutricional, podendo ser utilizados isolados ou combinado a outros parâmetros. ${ }^{8,9}$ Dentre os diversos protocolos de triagem de risco de desnutrição validados na literatura ressaltam-se a Mini Avaliação Nutricional (Mini Nutrition Assessment - MNA), Mini Avaliação Nutricional Simplificada (Mini Nutrition Assessment Short Form - MNA-SF), Avaliação Subjetiva Global (Subjective Global Assessment ASG), Triagem de Risco Nutricional (Nutritional Risk Screening - NRS 2002) e Instrumento Universal de Triagem de Desnutrição (Malnutrition Universal Screening Tool-MUST). ${ }^{10-14}$

Conforme o protocolo aplicado, poderão ser avaliados dados de peso, perda de peso não intencional, história nutricional, ingestão alimentar e hídrica, sintomas gastrointestinais, capacidade funcional, exame físico e diagnóstico de doenças, propiciando assim um diagnóstico nutricional baseado em várias informações, diferentemente do índice de massa corporal (IMC) que avalia apenas proporção do peso para a altura. ${ }^{10-15}$ Esses protocolos visam à detecção precoce de desnutrição, por isso sua aplicação deverá ocorrer nas primeiras horas de internação, para que o manejo nutricional inicie-se rapidamente, contribuindo com a melhora do estado nutricional do indivíduo, facilitando a sua melhora ou cura e, consequentemente, ocasionando a diminuição de despesas relacionadas à internações hospitalares. ${ }^{16,17}$

Em consonância às recentes recomendações do Ministério da Saúde, o presente estudo teve como objetivo verificar a prevalência de desnutrição e de risco nutricional em indivíduos hospitalizados e a concordância do diagnóstico nutricional através de cinco protocolos de triagem nutricional.

\section{MÉTODOS}

Foi realizado um estudo transversal no Hospital São José, um hospital de pequeno porte e de baixa complexidade, destinado ao atendimento de pacientes do Sistema Único de Saúde, convênios e particulares, na cidade de Arroio do Meio, Rio Grande do Sul. Utilizou-se a amostragem não probabilística por conveniência e a coleta de dados aconteceu entre janeiro e maio de 2013. O estudo foi aprovado pelo Comitê de Ética da Univates, sob protocolo número 043282/2012. Os pacientes foram convidados a participar da pesquisa em seus próprios leitos. Após o consentimento da participação através da assinatura do termo de consentimento livre e esclarecido, os pacientes foram submetidos à aplicação dos cinco protocolos.

Foram incluídos indivíduos de ambos os gêneros e com idade superior a 18 anos. Os critérios de exclusão foram: deficiência mental e/ou alterações psicológicas, dependência química por entorpecentes, gestação, presença de ascite e/ou edema e reinternação no período da coleta de dados. Para a coleta dos dados foram realizadas entrevistas durante as primeiras 48 horas de admissão ao hospital, quando foram coletados os dados de idade, antropometria e tempo de internação hospitalar até o momento, e foram aplicados cinco protocolos de triagem nutricional. A informação sobre o tempo de internação total foi obtida no momento da alta hospitalar, diretamente no prontuário dos pacientes. 
Os dados de peso e altura foram obtidos dos prontuários clínicos, sendo esta aferição realizada rotineiramente por técnicos de enfermagem com auxílio da balança da marca $\mathrm{Welmi}^{\circledR}$, com precisão de $100 \mathrm{~g}$ e capacidade de $150 \mathrm{~kg}$, no momento da internação. A altura foi medida com o estadiômetro acoplado à balança supracitada.

A classificação do estado nutricional foi realizada através do IMC, sendo utilizados os pontos de corte estabelecidos pela Organização Mundial da Saúde (OMS): IMC $<16,0 \mathrm{~kg} / \mathrm{m}^{2}$ magreza de grau III, IMC $16-16,9 \mathrm{~kg} / \mathrm{m}^{2}$ magreza de grau II, IMC $17,0-18,4 \mathrm{~kg} / \mathrm{m}^{2}$ magreza de grau I, IMC $18,5-24,9 \mathrm{~kg} / \mathrm{m}^{2}$ eutrofia, IMC $25-29,9 \mathrm{~kg} / \mathrm{m}^{2}$ pré-obesidade, IMC $30,0-34,9 \mathrm{~kg} / \mathrm{m}^{2}$ obesidade grau I, IMC $35,0-39,9 \mathrm{~kg} / \mathrm{m}^{2}$ obesidade grau II e IMC $>40 \mathrm{~kg} / \mathrm{m}^{2}$ obesidade grau III. ${ }^{15}$ Para os indivíduos idosos utilizaram-se os parâmetros recomendados por Lipschitz: IMC $<22 \mathrm{~kg} / \mathrm{m}^{2}$ classifica o indivíduo com desnutrição, IMC $22-27 \mathrm{~kg} / \mathrm{m}^{2} \mathrm{com}$ eutrofia e IMC $>27 \mathrm{~kg} / \mathrm{m}^{2}$ com excesso de peso. ${ }^{18}$

Os protocolos de triagem nutricional selecionados foram: MNA, MNA-SF, ASG, NRS 2002 e MUST. O protocolo MNA foi criado para ser aplicado em idosos, entretanto atualmente é também utilizado em adultos não idosos, com questões que contemplam alimentação, aspectos físicos e mentais. Os valores de referência do MNA foram: 24 a 30 pontos estado nutricional normal, 17 a 23,5 pontos risco de desnutrição e a pontuação menor de 17 pontos desnutrição, composta por questões variadas como peso, perda de peso nos últimos três meses, modo de se alimentar, quantidade de água ingerida, ingestão de frutas, verduras e carne. ${ }^{10}$ Já o protocolo MNA-SF é a forma reduzida do MNA, sendo composto somente pela primeira parte do protocolo original. Os valores de referência para diagnóstico nutricional são iguais aos do MNA. ${ }^{11}$

ASG é um método sistemático para a obtenção da história nutricional, exame físico e aplicação de avaliação clínica para verificação do estado nutricional, através da avaliação da perda de peso involuntária, ingestão alimentar habitual, sintomas gastrintestinais, doenças, exame físico e capacidade funcional. Os valores de referência são: pontuação menor de 17 pontos, estado de boa nutrição; entre 17 a 22 pontos, estado de desnutrição leve/moderado; e pontuação maior de 22 pontos, desnutrição grave. ${ }^{12}$

O protocolo NRS 2002 foi desenvolvido para aplicação hospitalar, sendo composto por cinco itens: IMC, perda de peso não intencional no período de três meses, alterações na absorção e na ingestão dos alimentos e alterações da própria doença. Os valores de referência são: pontuação $>3$ caracteriza risco nutricional e recomenda-se cuidado nutricional; pontuação $<3$ recomenda-se reavaliar o paciente semanalmente e, além disso, se o paciente tiver idade superior a 70 anos, deverá ser acrescentado um ponto no total da avaliação. ${ }^{13}$

O instrumento MUST é composto por três critérios, sendo eles: IMC, perda de peso não intencional nos últimos três a seis meses e presença de doenças agudas ou jejum prolongado por mais de cinco dias. Os valores de referência são: pontuação 0 , define baixo risco nutricional, 1 ponto define médio risco nutricional e $\geq 2$ pontos define alto risco nutricional. ${ }^{14}$

A ordenação dos protocolos foi estabelecida pela pesquisadora. Primeiramente optou-se pelo MNA, em seguida MNA-SF, ASG, NRS 2002 e por fim o protocolo MUST. Todos os protocolos foram aplicados pela pesquisadora no momento da entrevista, seguindo esta ordem. A pesquisadora foi previamente treinada para a aplicação desses protocolos.

Para a análise estatística de concordância entre os protocolos utilizou-se a dicotomização das classes dos escores da avaliação. A classificação quanto ao risco nutricional, desnutrição e bem nutrido, foram convertidas em duas classes. Essa conversão realizou-se com o total da pontuação de cada escore: pontuação de MNA $0=$ bem nutrido e $1=$ desnutridos (contemplando risco de desnutrição 1 e 2 desnutrido); MNA SF $0=$ bem nutrido e 1 = desnutridos (contemplando risco de desnutrição 1 e 2 desnutrido); MUST $0=$ bem nutrido (ou baixo risco 0 ) e $1=$ desnutridos (contemplando risco médio 1 e 2 alto risco); ASG $0=$ bem nutrido e 1 = desnutridos (contemplando desnutrição moderada e desnutrição grave); NRS $0=$ bem nutrido (reavaliação semanal) e $1=$ desnutridos (contemplando risco nutricional 2).

Para a análise estatística, as variáveis quantitativas foram descritas por média e desvio padrão, e as variáveis qualitativas foram descritas por percentuais. Para avaliar a concordância entre os protocolos, foi aplicado o coeficiente Kappa em conjunto com o intervalo de $95 \%$ de confiança, e para avaliação da associação entre tempo de internação e prevalência de risco nutricional, foi aplicado o teste qui-quadrado de tendência linear. $\mathrm{O}$ nível de significância adotado foi de $5 \%(\mathrm{p} \leq 0,05)$ e as análises foram realizadas no programa Statistical Package for the Social Sciences (SPSS), na versão 18.0.

\section{RESULTADOS}

Foram convidados a participar da pesquisa 105 indivíduos e, destes, 100 aceitaram participar do estudo 
Tabela 1. Avaliação da concordância entre cinco protocolos de triagem nutricional em 100 pacientes internados em um hospital de baixa complexidade de uma cidade do interior do Rio Grande do Sul, Brasil.

\begin{tabular}{lccccc|}
\multicolumn{1}{c}{$\begin{array}{c}\text { Protocolos de } \\
\text { triagem nutricional }\end{array}$} & $\begin{array}{c}\text { Prevalência de } \\
\text { risco nutricional }\end{array}$ & Kappa* & IC $\mathbf{9 5 \%}$ & $\begin{array}{c}\text { Concordância } \\
\text { (\%) }\end{array}$ \\
\hline MNA x MNA-SF & $49 \%$ vs $53 \%$ & 0,760 & 0,63 a 0,89 & $<0,001$ & 88 \\
\hline MNA x MUST & $49 \%$ vs $23 \%$ & 0,110 & $-0,06$ a 0,28 & 0,194 & 56 \\
\hline MNA x ASG & $49 \%$ vs $4 \%$ & 0,042 & $-0,04$ a 0,12 & 0,288 & 53 \\
\hline MNA x NRS & $49 \%$ vs $7 \%$ & 0,145 & 0,04 a 0,25 & 0,005 & 58 \\
\hline MNA-SF x MUST & $53 \%$ vs $23 \%$ & 0,148 & $-0,01$ a 0,30 & 0,070 & 56 \\
\hline MNA-SF x ASG & $53 \%$ vs $4 \%$ & 0,033 & $-0,04$ a 0,10 & 0,368 & 49 \\
\hline MNA-SF x NRS & $53 \%$ vs $7 \%$ & 0,125 & 0,04 a 0,22 & 0,010 & 54 \\
\hline MUST x ASG & $23 \%$ vs $4 \%$ & 0,165 & $-0,02$ a 0,35 & 0,012 & 79 \\
\hline MUST x NRS & $23 \%$ vs $7 \%$ & 0,328 & 0,11 a 0,54 & $<0,001$ & 82 \\
\hline ASG $\times$ NRS & $4 \%$ vs $7 \%$ & 0,330 & $-0,04$ a 0,70 & 0,001 & 93 \\
\hline
\end{tabular}

* Coeficiente de concordância de Kappa.

IC: Intervalo de confiança.

MNA: Mini Nutrition Assessment (Mini Avaliação Nutricional); MNA-SF: Mini Nutrition Assessment Short Form (Mini Avaliação Nutricional Simplificada); ASG: Subjective Global Assessment (Avaliação Subjetiva Global); NRS: Nutritional Risk Screening (Triagem para Risco Nutricional); MUST: Malnutrition Universal Screening Tool (Instrumento Universal de Triagem de Desnutrição).

e compuseram a amostra. A idade média foi de $62,2 \pm 19,2$ anos e 77 indivíduos (77\%) eram do gênero feminino. $\mathrm{Na}$ avaliação nutricional através do IMC, observou-se que a média foi de $27,0 \pm 5,8 \mathrm{~kg} / \mathrm{m}^{2}$. Os resultados da classificação do IMC foram: 38 (38\%) eram eutróficos, 29 (29\%) com excesso de peso/sobrepeso, 18 (18\%) obesos de grau I, 11 (11\%) obesos de grau II, um (1\%) obeso de grau III, dois ( $2 \%$ ) magreza de grau I e um (1\%) magreza de grau II. Sobre o tempo de internação, verificou-se que $78(78 \%)$ indivíduos permaneceram menos de três dias hospitalizados, $16(16 \%)$ entre três e sete dias hospitalizados e seis $(6 \%)$ mais de sete dias de internação.

$\mathrm{Na}$ avaliação do risco nutricional, observou-se que 16 homens e 33 mulheres (49\% dos pacientes) apresentaram risco com o protocolo MNA, 17 homens e 36 mulheres ( $53 \%$ dos pacientes) com MNA-SF, oito homens e 15 mulheres ( $23 \%$ dos pacientes) com MUST, dois homens e cinco mulheres ( $7 \%$ dos pacientes) com NRS, e um homem e três mulheres ( $4 \%$ dos pacientes) com ASG.

Quando testada a concordância entre os protocolos de triagem nutricional, verificou-se que praticamente todas as concordâncias foram fracas, exceto entre os protocolos da MNA e MNA-SF, em que houve uma boa concordância $(\mathrm{k}=0,760)$ (Tabela 1).

Não houve relação entre tempo de internação e risco nutricional em nenhum dos protocolos (Tabela 2). Investigando a relação do risco nutricional com a idade dos pacientes, três dos protocolos, MNA-SF, MUST e NRS, detectaram maior risco nutricional em pacientes com mais de 60 anos, considerando o nível de significância em $\mathrm{p} \leq 0,05$ (Tabela 3 ).
Tabela 2. Avaliação do percentual de risco nutricional conforme tempo de internação em 100 pacientes internados em um hospital de baixa complexidade de uma cidade do interior do Rio Grande do Sul, Brasil.

\begin{tabular}{|c|c|c|c|c|}
\hline \multirow[b]{2}{*}{ Protocolos } & \multicolumn{3}{|c|}{ Dias de internação } & \multirow[b]{2}{*}{$\mathbf{p}^{*}$} \\
\hline & $\begin{array}{c}£ 3 \\
(\mathrm{n}=78) \\
\mathrm{n}(\%)\end{array}$ & $\begin{array}{c}4-7 \\
(n=16) \\
n(\%)\end{array}$ & $\begin{array}{c}>7 \\
(n=6) \\
n(\%)\end{array}$ & \\
\hline MNA & $36(46,2)$ & $10(62,5)$ & $3(50,0)$ & 0,542 \\
\hline MNA-SF & $38(48,7)$ & $11(68,8)$ & $4(66,7)$ & 0,194 \\
\hline MUST & $16(20,5)$ & $5(31,3)$ & $2(33,3)$ & 0,325 \\
\hline ASG & $4(5,1)$ & $0(0,0)$ & $0(0,0)$ & 0,366 \\
\hline NRS & $5(6,4)$ & $1(6,3)$ & $1(16,7)$ & 0,409 \\
\hline
\end{tabular}

* Teste qui-quadrado.

MNA: Mini Nutrition Assessment (Mini Avaliação Nutricional). MNA-SF. Mini Nutrition Assessment Short Form (Mini Avaliação Nutricional Simplificada); ASG: Subjective Global Assessment (Avaliação Subjetiva Global); NRS: Nutritional Risk Screening (Triagem para Risco Nutricional); MUST: Malnutrition Universal Screening Tool (Instrumento Universal de Triagem de Desnutrição).

Tabela 3. Avaliação do percentual de risco nutricional conforme faixa etária em 100 pacientes internados em um hospital de baixa complexidade de uma cidade do interior do Rio Grande do Sul, Brasil.

\begin{tabular}{|c|c|c|c|}
\hline \multirow[b]{2}{*}{ Protocolos } & \multicolumn{2}{|c|}{ Faixa etária } & \multirow[b]{2}{*}{ p } \\
\hline & $\begin{array}{c}<60 \text { anos } \\
(n=46) \\
n(\%)\end{array}$ & $\begin{array}{c}>60 \text { anos } \\
(n=54) \\
n(\%)\end{array}$ & \\
\hline MNA & $20(43,5)$ & $29(53,7)$ & $0,413^{*}$ \\
\hline MNA-SF & $19(41,3)$ & $34(63,0)$ & $0,050^{*}$ \\
\hline MUST & $5(10,9)$ & $18(33,3)$ & $0,015^{*}$ \\
\hline ASG & $1(2,2)$ & $3(5,6)$ & $0,622^{* *}$ \\
\hline NRS & $0(0,0)$ & $7(13,0)$ & $0,014^{* *}$ \\
\hline
\end{tabular}

* Teste qui-quadrado; ** Teste exato de Fisher. Nível de significância: $p \leq 0,05$ IC: Intervalo de confiança.

MNA: Mini Nutrition Assessment (Mini Avaliação Nutricional); MNA-SF: Mini Nutrition Assessment Short Form (Mini Avaliação Nutricional Simplificada); ASG: Subjective Global Assessment (Avaliação Subjetiva Global); NRS: Nutritional Risk Screening (Triagem para Risco Nutricional); MUST: Malnutrition Universal Screening Tool (Instrumento Universal de Triagem de Desnutrição) 


\section{DISCUSSÃO}

No presente estudo, constatou-se que indivíduos de maior idade apresentaram maior percentual de risco nutricional. Na avaliação da concordância entre os protocolos de triagem nutricional observou-se que a MNA e MNA-SF foram os protocolos que obtiveram melhor concordância. Os demais protocolos utilizados não apresentaram boa concordância, provavelmente devido às diferentes variáveis avaliadas por cada protocolo.

O protocolo da MNA-SF foi desenvolvido a partir da MNA, com objetivo de diminuir o tempo de aplicação. O protocolo da MNA-SF contém seis questões, sendo utilizada somente a primeira parte do protocolo original, justificando-se assim a semelhança e concordância entre os dois protocolos, ao contrário dos demais protocolos utilizados. ${ }^{19}$ Assim como este estudo, outros encontraram boa concordância e correlação entre os protocolos MNA e MNA-SF. ${ }^{20,21}$

A prevalência de desnutrição pela MNA observada no presente estudo foi superior aos percentuais obtidos por outros autores. Um estudo realizado em 38 hospitais, com 1.000 indivíduos avaliados pelo protocolo da MNA, verificou que $52,4 \%$ eram bem nutridos, 36,1\% moderadamente desnutridos e $1,2 \%$ desnutridos. ${ }^{20}$ Paz et al..$^{21}$ descreveram que $33,3 \%$ dos indivíduos avaliados apresentavam desnutrição pela MNA.

Barbosa avaliou 705 indivíduos com o objetivo de verificar as variações na detecção do risco entre os protocolos de triagem nutricional de MUST, NRS, MNA-SF e ASG. Observando os resultados do referido estudo, pode-se verificar que o número de pacientes com risco de desnutrição foi de $279(39,6 \%)$ de acordo com MUST, 516 (73,2\%) com MNA-SF, 197 (27,9\%) com NRS e $274(38,9 \%)$ com ASG. ${ }^{22}$ Estes resultados são semelhantes aos observados no presente estudo no sentido de haver variações na detecção de risco nutricional conforme o protocolo empregado.

Para o protocolo de NRS, Kyle et al. ${ }^{23}$ estudaram 995 indivíduos e observaram uma maior especificidade no protocolo de NRS para a detecção de risco nutricional, quando comparado ao MUST, em período de internação superior a oito dias. Com a utilização desse mesmo protocolo, Bauer et al. ${ }^{24}$ observaram risco nutricional em $40,3 \%$ dos indivíduos avaliados. No presente estudo verificou-se $23 \%$ de risco nutricional através de NRS, valores inferiores quando comparados com as referidas pesquisas.

Em outro estudo, com população similar, foi observado risco nutricional em $6,1 \%$ dos indivíduos com o protocolo de NRS, sendo estes resultados semelhantes ao presente estudo. ${ }^{25}$ Stratton et al. ${ }^{26}$ avaliaram a concordância entre os protocolos de NRS e MUST, tendo observado ótima concordância entre eles, diferentemente dos achados no presente estudo. Quanto à avaliação de risco nutricional pela ASG, a prevalência de desnutrição foi observada em $48,1 \%$ da amostra do estudo IBRANUTRI ${ }^{2}$ e em $45 \%$ dos indivíduos avaliados por Naber et al., ${ }^{27}$ contrapondo-se aos resultados do presente estudo.

Realizando uma revisão da literatura sobre avaliação nutricional subjetiva, Barbosa-Silva e Barros ${ }^{28}$ sugerem que esta pode ser aplicada em pacientes cirúrgicos e também em diversas áreas clínicas. Em sua revisão, encontraram que com pequenas modificações para cada situação clínica, os protocolos de avaliação nutricional geralmente conseguem identificar os pacientes com maior risco nutricional. ${ }^{28}$ Venzin et al. ${ }^{29}$ observaram uma relação entre o período de internação e gravidade da patologia, não havendo a mesma relação quando comparado ao estado nutricional. King et al. ${ }^{30} \mathrm{e}$ Ozkalkanli et al. ${ }^{31}$ verificaram que o protocolo MUST foi capaz de predizer melhor o período de internação dos indivíduos, quando comparado aos protocolos MNA-SF e ASG, resultado que não foi observado no presente estudo. Ainda no estudo de King et al., ${ }^{30}$ pode-se observar que os indivíduos avaliados foram idosos e pacientes submetidos a tratamentos ortopédicos, diferenciando-se deste estudo, o qual abordou uma população heterogênea.

De acordo com estudo realizado por Arellano et al., ${ }^{32}$ os indivíduos com idade superior a 70 anos são os mais propensos ao desenvolvimento de risco nutricional, sendo a prevalência de risco entre 15 a $60 \%$ dentre eles. No presente estudo, os dados encontrados corroboram com a pesquisa citada. Já Bezerra et al., ${ }^{33}$ em estudo comparativo entre os protocolos de triagem nutricional, não observaram diferença estatisticamente significativa no risco nutricional entre adultos e idosos, segundo os protocolos NRS e MUST; resultados opostos aos achados do presente estudo, onde os indivíduos idosos apresentaram maiores percentuais de risco nutricional pela NRS e MUST, quando comparados aos adultos.

Vale ressaltar que neste estudo foram encontradas algumas limitações, dentre elas, o período de permanência hospitalar, onde a maioria das doenças diagnosticadas era de baixa complexidade, não exigindo mais do que três dias de internação para a recuperação. Os dados antropométricos foram retirados dos prontuários clínicos onde eram aferidos no momento da internação, porém esta limitação 
foi minimizada através da utilização da mesma metodologia de obtenção das medidas antropométricas para todos os protocolos, assim diminuindo o viés de aferição e não interferindo na análise de comparação entre os mesmos.

Os resultados obtidos sugerem que houve uma boa concordância entre os protocolos de MNA e MNA-SF. Este resultado seria esperado, uma vez que estes avaliam variáveis semelhantes. Nos demais protocolos, entretanto, não foi observada essa mesma concordância, provavelmente devido ao número e ao tipo de variáveis avaliadas serem diferentes em cada um deles.

Os pacientes idosos apresentaram risco nutricional pelos protocolos MNA-SF, MUST e NRS. Nestes protocolos são avaliados o IMC, perda de peso, ingestão alimentar, patologias diagnosticadas e idade, sendo essas variáveis facilmente alteradas em idosos hospitalizados. Na avaliação do estado nutricional realizada através do IMC verificou-se predomínio de sobrepeso e obesidade. Esses pacientes são considerados bem nutridos pelos protocolos de triagem nutricional, que buscam identificar especialmente os pacientes em risco de desnutrição.

Por fim, salientamos que para a escolha do protocolo de triagem nutricional a ser utilizado em indivíduos hospitalizados, torna-se necessário considerar o local e o perfil dos pacientes a serem avaliados, para que estes recebam a atenção nutricional apropriada.

\section{AGRADECIMENTOS}

Agradecemos às nutricionistas Janete Brod e Juliana Scheibler, responsáveis pelo Serviço de Nutrição e Dietética do Hospital São José, por destinar seu tempo à viabilização desta pesquisa; e ao diretor do Hospital, José Clovis Soares, que colaborou com a coleta de dados.

\section{REFERÊNCIAS}

1. Kondrup J, Johansen N, Plum LM, Bak L, Larsen IH, Martinsen A, Andersen JR, Baernthsen H, Bunch E, Lauesen N. Incidence of nutritional risk and causes of inadequate nutritional care in hospitals. Clin Nutr. 2002;21(6):461-8.

2. Waitzberg DL, Caiaffa WT, Correia MI. Hospital malnutrition: the Brazilian national survey (IBRANUTRI): a study of 4000 patients. Nutrition. 2001;17(7-8):e573-80

3. Brasil. Ministério da Saúde. Portaria n. 343/GM, de 7 de março de 2005. Diário Oficial, Brasília, 8 mar. 2005. Artigo 5º p.1-1.

4. Beck AM, Balknas UN, Camilo ME, Furst P, Gentile MG, Hasunen K, Jones L, Jonkers-Schuitema C, Keller U, Melchior JC, Mikkelsen BE, Pavcic M, Schauder P, Sivonen L, Zinck O, Øien H, Ovesen L. Practices in relation to nutritional care and support-report from the Council of Europe. Clin Nutr. 2002;21(4):351-4.

5. Lochs H, Allison SP, Meier R, Pirlich M, Kondrup J, Schneider S, van den Berghe G, Pichard C. Introductory to the ESPEN Guidelines on Enteral Nutrition: Terminology, definitions and general topics. Clin Nutr. 2006;25(2):e180-6.

6. Norman K, Pichard C, Lochs H, Pirlich M. Prognostic impact of disease-related malnutrition. Clin Nutr. 2008; 27(1):e5-15.

7. Kruizenga HM, Van Tulder MW, Seidell JC, Thijs A, Ader HJ, Van Bokhorst-de van der Schueren MAE. Effectiveness and cost-effectiveness of early screening and treatment of malnourished patients. Am J Clin Nutr. 2005; 82(5):1082-9.

8. Agarwal E, Ferguson M, Banks M, Batterham M, Bauer J, Capra S, Isenring E. Nutrition care practices in hospital wards: Results from the Nutrition Care Day Survey 2010. Clin Nutr. 2012;31(6):995-1001.

9. Van Bokhorst-de van der Schueren MAE, Guaitoli PR, Jansma EP, de Vet HCW. Nutrition screening tolls: Does one size fit all? A systematic review of screening tools for the hospital setting. Clin Nutr. 2014;33(1):39-58.

10. Guigoz Y, Vellas B, Garry PJ. Assessing the nutritional status of the elderly: The Mini Nutritional Assessment as part of the geriatric evaluation. Nutr Rev. 1996;54(1):S59-65.

11. Vellas B, Villars H, Abellan G, Soto ME, Rolland,Y, Guigz Y, Morley JE, Chumlea W, Salva A, Rubenstein LZ, Garry P. Overview of the MNA - Its history and challenges. J Nutr Health and Aging. 2006;10(6):456-63.

12. Detsky AS, Baker JP, O’Rourke K, Johnston N, Whitwell J, Mendelson RA, Jeejeebhoy KN. Predicting nutrition-associated complications for patients undergoing gastrointestinal surgery. J Parenter Enteral Nutr. 1987;11(5):e440-6.

13. Kondrup J, Rasmussen HH, Hamberg O, Stanga Z, Ad Hoc ESPEN Working Group. Nutritional risk screening (NRS 2002): a new method based on an analysis of controlled clinical trials. Clin Nutr. 2003;22(3):e321-36.

14. Stratton RJ, Hackston A, Longmore D, Dixon R, Price S, Stroud M, King C, Elia M. Malnutrition in hospital outpatients and inpatients: prevalence, concurrent validity and ease of use of the "malnutrition universal screening tool" ("MUST") for adults. Br J Nutr. 2004;92(5):e799=808.

15. World Health Organization. Obesity: Preventing and managing the global epidemic. Report of a WHO Expert Committee. World Health Organ Tech Rep Ser. 2000:1-252. [citado 05 de agosto de 2013]. Disponível em: http://whqlibdoc.who.int/trs/WHO_TRS_894.pdf

16. St-Arnaud-McKenzie D, Paquet C, Kergoat MJ, Ferland G, Dubé L. Hunger and aversion: drives that influence food intake of hospitalized geriatric patients. J Gerontol A Biol Sci Med Sci. 2004;59(12):e130-9.

17. Garcia RS, Tavares LR, Pastore CA. Nutrition screening in surgical patients of a teaching hospital from Southern Brazil: the impact of nutritional risk in clinical outcomes. Einstein. 2013;11(2):147-52. 
18. Lipschitz DA. Screening for nutritional status in the elderly. Prim Care. 1994;21(1):55-67.

19. Rubenstein LZ, Harker JO, Salvà A, Guigoz Y, Vellas B. Screening for undernutrition in geriatric practice: developing the short-form mini-nutritional assessment (MNA-SF). J Gerontol A Biol Sci Med Sci. 2001;56(6):eM366-72.

20. Argentina. Asociación Argentina de Nutrición Enteral y Parenteral. Estudio A.A.N.E.P. 99: prevalencia de desnutrición en hospitales de la Argentina [Internet]. Argentina; 2001 [citado 05 de agosto de 2013]. Disponível em: http://www.aanep.org.ar/prof_estudio99_02.php

21. Paz RC, Fazzio DMG, Santos ALB. Avaliação nutricional em idosos institucionalizados. Revisa. 2012;1(1):9-18.

22. Barbosa, MRP. Desempenho de testes de rastreamento e avaliação nutricional com preditores e desfechos clínicos negativos em pacientes hospitalizados [tese]. [São Paulo]: Universidade de São Paulo; 2010. 115 p.

23. Kyle UG, Kossovsky MP, Karsegard VL, Pichard C. Comparison of tools for nutritional assessment and screening at hospital admission: a population study. Clin Nutr. 2006;25(3):e409-17.

24. Bauer JM, Vogl T, Wicklein S, Trogner J, Muhlberg W, Sieber CC. Comparison of the Mini Nutritional Assessment, Subjective Global Assessment, and Nutritional Risk Screening (NRS 2002) for nutritional screening and assessment in geriatric hospital patients. Z Gerontol Geriatr. 2005;38(5):e322-7.

25. Gur AS, Atahan K, Aladag I, Durak E, Cokmez A, Tarcan E, Tavusbay C. The efficacy of the nutrition risk screening-2002 (NRS-2002) to decide on the nutritional support in general surgery patients. Bratisl Lek Listy. 2009;110(5):290-2.

26. Stratton RJ, King CL, Stroud MA, Jackson AA, Elia M. "Malnutrition Universal Screening Tool” predicts mortality and length of hospital stay in acutely ill elderly. Br J Nutr. 2006;95(2):325-30.

27. Naber THJ, Schernmer T, Bree A, Nusteling K, Eggink L, Kruimel JW, Bakkeren J, van Heereveld H, Katan MB. Prevalence of malnutrition in nonsurgical hospitalized patients and its association with disease complication. Am J Clin Nutr. 1997;66(5):1232-9.

28. Barbosa-Silva MCG, Barros AJD. Avaliação nutricional subjetiva: Parte 2 - revisão de suas adaptações e utilizações nas diversas especialidades clínicas. Arq Gastroenterol. 2002;39(4):248-52.

29. Venzin RM, Kamber N, Keller WC, Suter PM, Reinhart WH. How important is malnutrition? A prospective study in internal medicine. Eur J Clin Nutr. 2009;63(3):e430-6.

30. King CL, Elia M, Stroud MA, Stratton RJ. The predictive validity of the malnutrition universal screening tool (MUST) with regard to mortality and length of stay in elderly inpatients. Clin Nutr. 2003;22(1):eS4.

31. Ozkalkanli MY, Ozkalkanli DT, Katircioglu K, Savaci S. Comparison of tools for nutrition assessment and screening for predicting the development of complications in orthopedic surgery. Nutr Clin Pract. 2009;24(2):e274-80.

32. Arellano M, Garcia-Caselles MP, Pi-Figueras M, Miralles R, Torres RM, Aguilera A, Cervera AM. Clinical impact of different scores of the mini nutritional assessment (MNA) in the diagnosis of malnutrition in patients with cognitive impairment. Arch Gerontol Geriatr Suppl. 2004;9:27-31.

33. Bezerra JD, Dantas MAM, Vale SHL, Dantas MMG, Leite LD. Aplicação de instrumentos de triagem nutricional em hospital geral: um estudo comparativo. Ciência \& Saúde. 2012;5(1):9-15. 